\title{
Interplay effects of carrier-envelope phase and plasmon resonances in terahertz generation by ionizing ultrashort optical pulses
}

\author{
I. V. Osovitskaya ${ }^{1,2}$, V. A. Kostin ${ }^{1,2}$, N. V. Vvedenskii ${ }^{1,2}$ \\ ${ }^{1}$ Institute of Applied Physics, Russian Academy of Sciences, Nizhny Novgorod, Russia, oivnn121@ mail.ru \\ ${ }^{2}$ University of Nizhny Novgorod, Nizhny Novgorod, Russia
}

The direct conversion of femtosecond ionizing laser pulses into low-frequency radiation attracts considerable attention due to associated possibilities for creating sources of powerful broadband terahertz $(\mathrm{THz})$ pulses. Previous studies of this phenomenon were mainly related to two limiting cases: (i) fewcycle (extremely short) ionizing pulses with several optical field cycles and (ii) multicycle (quasimonochromatic) pulses with a large number of field cycles. In the first case, the amplitude and energy of $\mathrm{THz}$ oscillations strongly depend on the carrier-envelope phase (CEP) in the ionizing pulse [1-3], whereas for multicycle pulses the CEP dependence is unnoticeable [4-6]. The spectral and mode structures of generated radiation also differ in these two cases. For the fewcycle pulses, the plasmonic oscillations corresponding to the dipole geometric resonance are excited and emitted from the laser-produced plasma filament to the surrounding space. For multicycle pulses, the radiation is produced by a relaxing current directed along the plasma filament, and the spectrum width and central frequency are determined by the collision frequency of electrons with heavy particles. Thus, the shape of low-frequency spectrum strongly depends on the pump pulse duration, and there is a range of durations where transition between different spectral shapes occurs. In this range of durations, the spectrum shape also significantly depends on the CEP, as was apparently observed in experiment [7]. Here, we study this intermediate case based on the universal model that considers the motion of the plasma electrons under forces both linear and quadratic with respect to the optical field strength.

The calculation of the $\mathrm{THz}$ radiation employs the two-stage approach [3, 5] which assumes that the characteristic frequency $f_{\mathrm{THz}}$ of the generated $\mathrm{THz}$ radiation is small compared with the inverse pump duration $1 / \tau$. $f_{\mathrm{THz}} \tau<<1$. During the short (femtosecond) first stage, a gas medium is ionized and the free-electron currents are excited in the produced plasma by the optical pump pulse. In the longer (picosecond) second stage, there is a self-consistent evolution of these free-electron currents and the electromagnetic fields generated by them. Such separation into stages is convenient since one can neglect lowfrequency plasma fields and assume the electric and magnetic fields given in the first stage, and one can consider fields and currents weak enough and neglect the nonlinear terms in the second stage.

Equations solved in the first stage are obtained directly from the kinetic equation

$$
\frac{\partial f}{\partial t}+\mathbf{v} \cdot \frac{\partial f}{\partial \mathbf{r}}-\frac{e}{m}\left(\mathbf{E}+\frac{\mathbf{v}}{c} \times \mathbf{B}\right) \cdot \frac{\partial f}{\partial \mathbf{v}}=I
$$

for the single-electron distribution function $f(\mathbf{r}, \mathbf{v}, t)$ (over positions $\mathbf{r}$ and velocities $\mathbf{v}$ ) with the ionization source term $I=\left(N_{m}-N\right) w_{\mathbf{v}}(\mathbf{E}, \mathbf{v})$. Here $e$ is the elementary charge, $m$ is the electron mass, $c$ is the speed of light, $\mathbf{E}(\mathbf{r}, t)$ and $\mathbf{B}(\mathbf{r}, t)$ are the electric and magnetic fields, $w_{\mathbf{v}}(\mathbf{E}, \mathbf{v})$ is the differential (with respect to the initial photoelectron velocities) ionization probability per unit time in the static field $\mathbf{E}, N_{m}$ is the initial density of neutral particles, and $N(\mathbf{r}, t)$ is the plasma density. Assuming the plasma being cold and the average velocity of newly-born electrons being zero, $\int w_{\mathbf{v}}(\mathbf{E}, \mathbf{v}) \mathbf{v} d^{3} \mathbf{v}=0$, one obtains equations for the plasma density, the electron density perturbation $n(\mathbf{r}, t)=\int f(\mathbf{r}, \mathbf{v}, t) d^{3} \mathbf{v}-N(\mathbf{r}, t)$, and the electron current density $\mathbf{j}(\mathbf{r}, t)=-e \int f(\mathbf{r}, \mathbf{v}, t) \mathbf{v} d^{3} \mathbf{v}$ as follows:

$$
\begin{gathered}
\frac{\partial N}{\partial t}=\left(N_{m}-N\right) w(\mathbf{E}), \frac{\partial n}{\partial t}=\frac{\nabla \cdot \mathbf{j}}{e}, \frac{\partial \mathbf{j}}{\partial t}=\frac{N e^{2}}{m} \mathbf{E}+\mathbf{S}, \\
\mathbf{S}=\frac{1}{e}\left[(\mathbf{j} \cdot \nabla) \frac{\mathbf{j}}{N+n}+\frac{\mathbf{j}}{N+n}(\nabla \cdot \mathbf{j})\right]+\frac{n e^{2}}{m} \mathbf{E}-\frac{e}{m c} \mathbf{j} \times \mathbf{B},(2)
\end{gathered}
$$

where $w(\mathbf{E})=\int w_{\mathbf{v}}(\mathbf{E}, \mathbf{v}) d^{3} \mathbf{v}$ is the ionization probability per unit time. For numerical calculations below, we use the atmospheric density value $N_{m}=3 \cdot 10^{19} \mathrm{~cm}^{-3}$ and the empirical formula for the hydrogen atom [8]:

$w(\mathbf{E})=4 \omega_{a}\left(E_{a} /|\mathbf{E}|\right) \exp \left(-2 E_{a} / 3|\mathbf{E}|-12|\mathbf{E}| / E_{a}\right)$, where $\omega_{a}=4.13 \cdot 10^{16} \mathrm{~s}^{-1}$ and $E_{a}=5.14 \cdot 10^{9} \mathrm{~V} / \mathrm{cm}$ are the atomic units of frequency and electric field. To find fields and currents, we develop the perturbation approach for Eqs. (1) and (2) assuming that $n<<N$ and all characteristic spatial scales (optical wavelength, size of plasma inhomogeneity) are large compared to the amplitude of electron oscillations.

In the first stage, we solve Eqs. (1) and (2) for the given fields $\mathbf{E}$ and $\mathbf{B}$ of the optical Bessel beam defined by the magnetic Hertz vector $\boldsymbol{\Pi}^{m}=\hat{\mathbf{y}} \int_{-\infty}^{\infty} A_{\omega} J_{0}\left(k_{\perp} r\right) e^{-i \omega \xi} d \omega / 2 \pi$, where $\theta$ is the focusing angle of the Bessel beam, $k_{\perp}=\sin \theta \omega / c$ is the transverse wavenumber, $\xi=t-z / V$ is the shifted time, $V=c / \cos \theta$ is the phase velocity, $r$ is the distance to the beam axis (the $z$ axis of the Cartesian and cylindrical coordinate systems), $J_{0}$ is the zero-order Bessel function of the first kind, $A_{\omega}$ is the Fourier spectrum of the $y$ component of the Hertz vector at the beam axis (since $E_{x}=c^{-2} \cos \theta d^{2} \Pi_{y}^{m} / d \xi^{2}$, the spectrum of $E_{x}$ at the axis is $\left.-\cos \theta(\omega / c)^{2} A_{\omega}\right)$. In numerical calculations, the temporal profile of the pulse at the axis is chosen as

$$
\begin{gathered}
\Pi_{y}^{m}=-\left[\left(8 \pi c^{3} I_{0}\right)^{1 / 2} /\left(\omega_{0} \cos \theta\right)^{2}\right] \sin ^{4}(\pi \xi / 2 \tau) \cos \left(\omega_{0} \xi+\psi\right) \\
\text { for }|\xi+\tau|<\tau, \Pi_{y}^{m}=0 \text { for }|\xi+\tau| \geq \tau \text {, where } \omega_{0} \text { is the }
\end{gathered}
$$


central frequency of the ionizing field (which corresponds to the wavelength $2 \pi c / \omega_{0}=800 \mathrm{~nm}$ in calculations), $I_{0}$ is the maximum field intensity at the axis, $\psi$ is the CEP. Thus, the fields depend on the shifted time $\xi$ and transverse coordinates (radial coordinate $r$ and azimuth angle $\varphi$ in the cylindrical coordinate system. Another simplification is related to the cylindrical symmetry of the beam: The calculations show that the plasma density depends weakly on $\varphi$ for small enough angles $\theta$, and this dependence can be neglected. The current density in the first order of perturbation theory has a dipole structure, i.e., its components in cylindrical coordinates have one azimuthal variation and are proportional to $\cos \varphi$ or $\sin \varphi$. The current density in the second order is a superposition of the symmetric term (the components do not depend on $\varphi$ ) and the term with two variations (components are proportional to $\cos 2 \varphi$ or $\sin 2 \varphi$ ).

We integrate the equations for the first and second orders inside the cylindrical area $r<a=$ $\mu_{0} c / \omega_{0} \sin \theta$, and determine the plasma density transverse profile remaining after the pump pulse has passed, $N_{R}(r)=N(r, \xi=0)$, and the residual current density (RCD) distribution $\mathbf{j}_{R}(r, \varphi)=\mathbf{j}(r, \varphi, \xi=0)$, which is a superposition of components with 0,1 , and 2 azimuthal variations; here $\mu_{0} \approx 2.405$ is the first zero of $J_{0}$. We assume the field is so weak outside this cylindrical domain that the plasma and currents produced there do not significant affect the generated radiation. Then, the calculated distributions are used as parameters and initial conditions for the second stage, where the Maxwell equations and the linear equation for the electron current density in a cold collision plasma are solved,

$$
\nabla \times \mathbf{E}=-\frac{1}{c} \frac{\partial \mathbf{B}}{\partial \xi}, \quad \nabla \times \mathbf{B}=\frac{1}{c} \frac{\partial \mathbf{E}}{\partial \xi}+\frac{4 \pi}{c} \mathbf{j}, \frac{\partial \mathbf{j}}{\partial \xi}+v \mathbf{j}=\frac{\omega_{p}^{2}}{4 \pi} \mathbf{E},
$$

where $\omega_{p}(r)=\left[4 \pi N_{R}(r) e^{2} / m\right]^{1 / 2}$ is the plasma frequency and $v$ is the collision frequency of electrons with heavy particles $\left(5 \cdot 10^{12} \mathrm{~s}^{-1}\right.$ in calculations $)$. The initial conditions for fields are $\left.\mathbf{E}\right|_{\xi=0}=0,\left.\mathbf{B}\right|_{\xi=0}=0$; the RCD found in the first stage serves as the initial condition for the electron current density, $\left.\mathbf{j}\right|_{\xi=0}=\mathbf{j}_{R}$. At the boundary $r=a$, the radiation conditions are set under assumption that there is no plasma in the external area, $N(r>a) \equiv 0$. We solve this initial problem separately for each of three azimuthal components of the RCD by applying the Laplace transform with respect to the variable $\xi$. After that, we calculate the spectral density $w_{\text {rad }}(f)$ and the radiated energy $W_{\text {rad }}=\int_{0}^{f_{\max }} w_{\text {rad }}(f) d f$ per unit length along the $z$ axis in $\mathrm{THz}$ range, where $f_{\max }$ is the upper bound of the $\mathrm{THz}$ range under consideration. The spectral density can be expressed in terms of the Laplace images of the longitudinal field components at the boundary of the computational area $w_{\mathrm{rad}}=w_{\mathrm{rad}}^{[0]}+w_{\mathrm{rad}}^{[1]}+w_{\mathrm{rad}}^{[2]}$, where

$$
w_{\mathrm{rad}}^{[M]}(f)=\frac{c^{2}}{2 \pi^{2} f \sin ^{2} \theta} \frac{\left|\widetilde{E}_{z}^{[M]}(a, 0)\right|^{2}+\left|\widetilde{B}_{z}^{[M]}(a, \pi / 2 M)\right|^{2}}{\left|H_{M}^{(1)}(2 \pi \sin \theta f a / c)\right|^{2}}
$$

is the spectral density of the radiated energy of the component with $M$ azimuthal variations, $\widetilde{E}_{z}^{[M]}(r, \varphi)$ and $\widetilde{B}_{z}^{[M]}(r, \varphi)$ are the Laplace images of the longitudinal components of the electric and magnetic fields, respectively, for the Laplace variable $p \rightarrow 2 \pi i f+0$, and $H_{M}^{(1)}$ is the Hankel function of the first kind and order $M$.

We solve numerically the above equations for a wide range of focusing angles $1^{\circ} \leq \theta \leq 30^{\circ}$, durations $4 \mathrm{fs} \leq \tau_{p}=\left[2 \arcsin \left(2^{-1 / 8}\right) / \pi\right] \tau \leq 50 \mathrm{fs}$, and intensities $10^{14} \mathrm{~W} / \mathrm{cm}^{2} \leq I_{0} \leq 10^{15} \mathrm{~W} / \mathrm{cm}^{2}$ of ionizing pulses for different CEPs $0 \leq \psi \leq \pi$. The dipole RCD strongly depends on $\psi$ for any pumps considered, whereas the distribution of the plasma density and the RCD terms with 0 and 2 azimuthal variations practically do not depend on $\psi$ even for extremely short durations $\tau_{p}$. Although the dipole RCD proves to be the weakest, for few-cycle pulses its contribution to $\mathrm{THz}$ radiation can be determinant. However, the dipole component decrease rapidly with increasing duration, and the contributions from different azimuthal components to $\mathrm{THz}$ radiation are already comparable at not too long durations. Under these conditions, the $\mathrm{THz}$ spectrum shape can vary greatly as $\psi$ changes. With a further increase in the duration, the dipole component becomes negligibly small, and the dependence on the CEP disappears.

This work was supported by the Russian Foundation for Basic Research (analytical study, Grants Nos. 16-32-60166, 18-02-01150 and 18-32-00951) and Russian Science Foundation (numerical calculations, Grant No. 18-11-00210).

\section{References}

1. Löffler, T., Jacob, F., Roskos, H. G. Generation of terahertz pulses by photo-ionization of air // Appl. Phys. Lett. 2000. V. 77, No. 3. P. 453-455.

2. D'Amico, C., Houard, A., Franco, M. Conical forward $\mathrm{THz}$ emission from femtosecond-laser-beam filamentation in air // Phys. Rev. Lett. 2007. V. 98, No. 23. P. 235002.

3. Kostin, V. A., Vvedenskii, N. V. Ionization-induced conversion of ultrashort Bessel beam to terahertz pulse // Opt. Lett. 2010. V. 35. No. 2. P. 247-249.

4. Kreß, M., Löffler, T., Thomson, M. D. Determination of the carrier-envelope phase of few-cycle laser pulses with terahertz-emission spectroscopy // Nat. Phys. 2006. V. 2, No. 5. P. 327-331.

5. Gildenburg, V. B., Vvedenskii, N. V. Optical-to-THz wave conversion via excitation of plasma oscillations in the tunneling-ionization process // Phys. Rev. Lett. 2007. V. 98, No. 24. P. 245002.

6. Silaev, A.A., Vvedenskii, N. V. Residual-current excitation in plasmas produced by few-cycle laser pulses // Phys. Rev. Lett. 2009. V. 102, No. 11. P. 115005.

7. Bai, Ya, Song, L., Xu, R., Li, C., Liu, P., Zeng, Z., Zhang, $Z$., $L u, H$. Waveform-controlled terahertz radiation from the air filament produced by few-cycle laser pulses // Phys. Rev. Lett. 2012. V. 108, No. 25. P. 255004.

8. Tong, X. M., Lin, C. D. Empirical formula for static field ionization rates of atoms and molecules by lasers in the barrier-suppression regime // J. Phys. B. 2005. V. 38. No. 15. P. 2593-2600. 